\section{Europe's biomarker push}

\section{By Mike Ward, Senior Editor}

European Commission officials are expected to unveil by the end of April the details of the first call for projects associated with the Commission's $€ 2$ billion ( $\$ 3.1$ billion) Innovative Medicines Initiative, which will attempt to establish industry-standard biomarkers across a range of therapeutic areas.

Although the Innovative Medicines Initiative (IMI) has disclosed some of its remit, details of the projects included in the first call for participation have not been officially released. ${ }^{1-3}$ But according to briefing materials prepared for stakeholder discussions and draft proposals seen on academic websites in Europe, the two largest programs are expected to cover safety biomarkers and immunogenicity of biotherapeutics.

IMI is a five-year, pan-European public and private collaboration that will involve large and small biopharma and healthcare companies, academics, regulators and patient groups. It has been established jointly by the European Commission (EC) and the European Federation of Pharmaceutical Industries and Associations (EFPIA) with the dual goal of enhancing the competitiveness of Europe's pharma industry and removing the principal causes of delay or precompetitive bottlenecks in R\&D.

According to IMI, 26 EFPIA members have signed up as potential participants.

Biotech companies, academic researchers, medical charities and regulatory agencies will be invited to establish so-called public consortia to work alongside pharma company consortia on topics initially selected by the EFPIA members and backed by funds from the EC's Framework Program 7 (FP7).

The first call will ask potential participants to express their interest by putting forward proposed consortia-with a proposed plan-that would address the challenges laid out in the programs identified by the pharma members and agreed to by the EC.

According to Ian Ragan, IMI advisor to EFPIA, the first call is likely to cover 18 research topics.

"We are working on publishing the first IMI call in April, although I am not sure if we will make it," Karima Boubekeur, head of external research and innovation environment at Roche, told SciBX. "There is an agreement in place on the topics to be addressed, which we expect the European Commission to announce in the Official Journal at the end of the month."

Although Boubekeur declined to reveal details of the first 18 programs, which were established following consultation with more than 150 participants from EFPIA, she was able to confirm that they would be worth a total of $€ 125$ million ( $\$ 193$ million) to successful applicants.

"The first call will have a total budget of $€ 250$ million, of which $€ 125$ million will come from FP7 while the pharmaceutical consortia will provide $€ 125$ million in kind," she said.

Under the IMI program, only research conducted in the European laboratories of multinational pharma companies will count toward the 'in-kind' contribution. "We have already established budget lines at Roche to track our IMI costs," said Boubekeur.

According to the unofficial program summaries, the 18 topics proposed by the pharma industry include 5 involving safety prediction, 1 pharmacovigilance project, 7 therapy-focused projects and 5 focusing on education and training.

According to the unofficial summaries, one of the largest proposalsprojected to be worth $€ 21$ million (\$32 million) over five years-would focus on the development of translational safety biomarkers from nonclinical to early clinical studies in three target organs: kidney, liver and the vascular system.

Proposed outputs could include a generic process for clinical validation of translational safety biomarkers, a set of biomarkers for drug-induced injuries and their assays-both qualified for use in early clinical trials - and a database with human biomarker profiles.

Another proposed program would focus on prediction and minimization of immunogenicity to biotherapeutics. With no reliable prediction methods for immunogenicity of therapeutic proteins, the proposed project would seek to standardize antidrug antibody analyses, to improve understanding of critical factors leading to immunogenicity and find ways to minimize them, and to optimize and harmonize existing preclinical tools.

The immunogenicity project would have up to $€ 13$ million ( $\$ 20$ million) in FP7 financing available to it.

Meanwhile, EFPIA members are said to have expressed a willingness to contribute about $€ 15$ million ( $\$ 23$ million) in kind to establish a program to develop new methods of pharmacovigilance and pharmacoepidemiology, such as signal identification and detection, and data mining based on large safety databases. That program also could last up to five years.

The first call also may include topics focusing on therapeutic approaches to diabetes, brain disorders and respiratory diseases, worth an estimated total of $€ 57$ million ( $\$ 88$ million).

To improve the predictivity of drug efficacy evaluation, a $€ 20$ million (\$31 million) five-year program would focus on the development of validated biomarkers and surrogate endpoints for micro- and macrovascular hard endpoints in diabetes clinical research. Identifying surrogate markers 
Table 1. Innovative Medicines Initiative principles on IP. European Federation of Pharmaceutical Industries and Associations members and the European Commission deliberated for two years to determine an IP policy for the Innovative Medicines Initiative (IMI) to achieve fair allocation of rights, reward innovation and broad participation of private and public entities in IMI projects. The policy covers IP, know-how and information generated and held by participants before the program and needed to carry out the projects ("background IP"), generated by the project participants in pursuit of project objectives ("foreground IP") or generated by the participants but outside of the project objectives ("sideground IP").

\begin{tabular}{lll}
\hline Issue & Background IP & Foreground IP \\
\hline Ownership & Each participant remains the exclusive owner. & $\begin{array}{l}\text { Participant that generated it. If several } \\
\text { participants: joint ownership. However, } \\
\text { a project agreement can allocate } \\
\text { differently. }\end{array}$ \\
\hline $\begin{array}{l}\text { Right for a participant to transfer its } \\
\text { ownership }\end{array}$ & $\begin{array}{l}\text { Free to transfer ownership, subject to rights and } \\
\text { obligations of the grant or project agreement } \\
\text { that is, the buyer accepts the same legal } \\
\text { project agreement. } \\
\text { position in relation to project). Notify other } \\
\text { participants after transfer. }\end{array}$ & $\begin{array}{l}\text { Each participant shall remain free to } \\
\text { license, assign or otherwise dispose of } \\
\text { its ownership rights. }\end{array}$ \\
\hline Rot applicable \\
\hline
\end{tabular}

Right for a participant to license, use and exploit independently of the other participants

Access rights for participants for the purposes of completing the project

Access rights for participants and affiliates for research use during the project or after the completion of the project

Access rights for third parties for research use after the completion of the project

Rights for participants (and affiliates) or third parties for direct exploitation after completion of the project participants after transfer.

Right to independently license nonexclusively and otherwise use.

Right to independently license nonexclusively and otherwise use.

Not applicable

Royalty-free and nonexclusive license solely for the purpose of participating in and completing the project.

Nonexclusive license on fair and reasonable terms or royalty free if needed for the research use of foreground IP generated by the project.

Requires nonexclusive license on fair and reasonable terms to needed for the research use of foreground IP generated by the project.

No access rights. Subject for commercial negotiation.
Royalty-free and nonexclusive license. Not applicable

Nonexclusive license of fair and reasonable terms or royalty free, as determined in the project agreement.

Nonexclusive license on fair and reasonable terms, as determined in the project agreement.

No access rights. Subject for commercial Not applicable negotiation.

\section{Source: Innovative Medicines Initiative}

could make studies into potential diabetes treatments shorter and less expensive.

Moreover, according to the unofficial draft material, IMI has acknowledged there also is a need to define mutually agreed surrogate endpoints that can be applied in clinical trials to test for treatment or prevention of microvascular complications.

According to the unofficial summaries, the first call also could provide some $€ 25$ million ( $\$ 39$ million) over five years to identify biomarkers and develop animal models for pain research and for psychiatric and neurodegenerative disorders.

Two proposed respiratory projects, focusing on identifying new targets and biomarkers for the treatment of both asthma and chronic obstructive pulmonary disease, could see more modest contributions of $€ 1$ million (\$1.5 million) each over five years.

According to Boubekeur, pharma companies already have established private consortia and resolved what each of them will contribute.

Biotech companies, academic groups and other stakeholders will have to establish their own public consortia and submit their proposals for contributing to the programs established by the pharma-EC agreement.

Public consortium proposals will be evaluated by a peer-review committee, assisted by ad hoc experts and EFPIA consortia members, to select a winning consortium for each topic. This process is expected to take three months from the publication of the first call.

The winning consortium will be invited to submit a full project proposal, written jointly with members of the EFPIA consortium, which will provide a full description of research activities, including responsibilities, budgets and timelines.
According to Boubekeur, a key part of the full project proposal will be a draft agreement covering how the partners will handle the IP generated by the programs.

As outlined on IMI's website, unless otherwise resolved as part of the project agreement, information and IP that participants bring into a project-so-called "background IP" - must be widely and readily available if it is necessary for the research use of IMI findings- "foreground IP."

Similarly, foreground IP must be readily available for research use during the program for participants and to third parties following its completion.

Results generated under the project, but outside the scope of the program objectives- "sideground IP"-belong to the participant that generated it (see Table 1, "Innovative Medicines Initiative principles on IP”).

To participate in a public consortium, potential members have to be independent legal entities with the capacity to carry out the work themselves. They can include academic teams, patient organizations and other not-for-profit legal entities.

To participate, companies must be small- to medium-sized enterprises as defined by the EU, employing fewer than 250 people and having an annual turnover not exceeding $€ 50$ million ( $\$ 77$ million) and/or an annual balance sheet of total assets not exceeding $€ 43$ million ( $\$ 66$ million).

Funds will only be provided for research performed in countries covered by the EU's FP7.

\section{REFERENCES}

1. Council of the European Union. Council regulation setting up the joint 


\section{PUBLIC-PRIVATE INTERFACE}

undertaking for the implementation of the joint technology initiative on innovative medicines. <http://www.imi-europe.org/DocStorage/ PublicSiteAdmin/Publications/FINAL_Legal_Package_18122007.pdf> (Dec. 18, 2007)

2. Innovative Medicines Initiative. The Innovative Medicines Initiative (IMI) strategic research agenda: creating biomedical R\&D leadership for europe to benefit patients and society. <http://www.imi-europe.org/DocStorage/ PublicSiteAdmin/Publications/Strategic\%20Research\%20Agenda\%20 (Version\%202).pdf> (Sept. 15, 2006)
3. Innovative Medicines Initiative. IMI intellectual property policy. <http:// www.imi-europe.org/DocStorage/PublicSiteAdmin/Publications/IMI_IPR_ policy_1_August_2007.pdf> (Aug. 1, 2007)

COMPANIES AND INSTITUTIONS MENTIONED

European Federation of Pharmaceutical Industries and Associations, Brussels, Belgium

Innovative Medicines Initiative, Brussels, Belgium

Roche (SWX:ROG), Basel, Switzerland 